Article

\title{
Smooth Muscle Cell Responses to Poly( $\varepsilon$-Caprolactone) Triacrylate Networks with Different Crosslinking Time
}

\author{
Jing Wang ${ }^{1}$, Li Liu ${ }^{2}$, Aoning Wang ${ }^{2}$, Xiang Liu ${ }^{1}$, Yi Zhang ${ }^{1, * \mathbb{D}}$, Zhoulu Wang ${ }^{1, *}$ and \\ Jinbo Dou $1,3, *$ \\ 1 School of Energy Sciences and Engineering, Nanjing Tech University, Nanjing 211816, China; \\ wjx2015@njtech.edu.cn (J.W.); iamxliu@njtech.edu.cn (X.L.) \\ 2 Key Laboratory of Flexible Electronics (KLOFE), Institute of Advanced Materials (IAM), \\ Nanjing Tech University, Nanjing 211816, China; 201861122099@njtech.edu.cn (L.L.); \\ aoningsmile@gmail.com (A.W.) \\ 3 Department of Materials Science and Engineering, The University of Tennessee, Knoxville, TN 37996, USA \\ * Correspondence: zhangy@njtech.edu.cn (Y.Z.); WangZhouLu66@163.com (Z.W.); jdou@vols.utk.edu (J.D.)
}

Received: 17 October 2020; Accepted: 23 November 2020; Published: 25 November 2020

\begin{abstract}
Poly( $\varepsilon$-caprolactone) triacrylate (PCLTA) is attractive in tissue engineering because of its good biocompatibility and processability. The crosslinking time strongly influences PCLTAs cellular behaviors. To investigate these influences, PCLTAs with different molecular weights were crosslinked under UV light for times ranging from 1 to $20 \mathrm{~min}$. The crosslinking efficiency of PCLTA increased with decreasing the molecular weight and increasing crosslinking time which could increase the gel fraction and network stiffness and decrease the swelling ratio. Then, the PCLTA networks crosslinked for different time were used as substrates for culturing rat aortic smooth muscle cells (SMCs). SMC attachment and proliferation all increased when the PCLTA molecular weight increased from $8 \mathrm{k}$ to $10 \mathrm{k}$ and then to $20 \mathrm{k}$ at the same crosslinking time. For the same PCLTA, SMC attachment, proliferation, and focal adhesions increased with increasing the crosslinking time, in particular, between the substrates crosslinked for less than $3 \mathrm{~min}$ and longer than $5 \mathrm{~min}$. This work will provide a good experimental basis for the application of PCLTA.
\end{abstract}

Keywords: poly(e-caprolactone) acrylates; crosslinking time; mechanical properties; smooth muscle cells

\section{Introduction}

Poly( $\varepsilon$-caprolactone) (PCL) is a biodegradable semi-crystalline polymer that has been widely used in tissue engineering [1-5]. The crystalline structure offers PCL unique thermal and mechanical properties. After the hydroxyl end groups in PCL diols or triols are converted into crosslinkable functional groups, i.e., acrylate, maleic anhydride, or fumarate, they can be crosslinked into networks with controllable chemical and physical properties [6-12]. Compared to the other crosslinking methods through heat, irradiation, or redox process, photo-crosslinking is more efficient to control the polymerization heat evolution and fabricate complex structures [13-16]. Wang et al. have developed photo-crosslinkable PCL acrylates (PCLAs) such as PCL diacrylates (PCLDAs) and PCL triacrylates (PCLTAs) [17]. Depending on the molecular weight of PCL precursor in the synthesis of PCLA, amorphous and semi-crystalline PCL networks were prepared with elastic modulus $(E)$ ranging from 1 to $200 \mathrm{MPa}$ and controllable melting temperature $\left(\mathrm{T}_{\mathrm{m}}\right)$ and crystallinity $\left(\chi_{\mathrm{c}}\right)$.

The crosslinks in the photo-crosslinked PCLAs suppressed and impeded the PCL crystallization, resulting in a reduced crystallinity. For photo-crosslinkable polymers, the crosslinking efficiency can be controlled by the density of crosslinkable groups, the concentration of photo-initiator, UV light 
intensity, and crosslinking time [18-22]. Among all these factors, sufficient crosslinking time should be given to ensure a polymer network with a high gel fraction and desirable mechanical properties without causing overcure and photo-scission problems [23,24]. For PCLAs, both crosslinking density and efficiency increase with increasing the density of acrylate end groups, which can be achieved by either decreasing the molecular weight of the PCL precursor with a certain architecture, or having multiple arms with more acrylate end groups in one polymer.

In this study, we studied the roles of crosslinking density and crystallinity in determining the physical properties, such as gel fraction, swelling ratio, and thermal, rheological, and mechanical properties, of photo-crosslinked PCLTA by varying both the crosslinking time and the molecular weight of the precursor. These PCLTA networks resulted from different crosslinking time were further used as substrates to regulate rat aortic smooth muscle cell (SMC) cell behaviors, including attachment, proliferation, spreading, and focal adhesion. This study demonstrates that photo-crosslinked PCLTA can not only promote SMC attachment, but also maintain focal adhesions, providing a new experimental scheme for the design and optimization of vascular tissue engineering scaffolds.

\section{Results and Discussion}

\subsection{Gel Fraction and Swelling Ratio}

Crosslinking kinetics is important for understanding how a polymer network is formed and when crosslinking is sufficient for a variety of properties. The gel fraction and swelling ratio in the $\mathrm{CH}_{2} \mathrm{Cl}_{2}$ of photo-crosslinked networks of the PCLTAs with different crosslinking times are shown in Figure 1. Because the composition of acrylate groups was lower in PCLTA with a higher molecular weight, the gel fraction decreased from PCLTA8k to PCLTA10k and then PCLTA20k, at the same crosslinking time. For the same PCLTA, the gel fraction increased asymptotically with increasing the crosslinking time. The distance between two neighboring crosslinks in the network was determined by both the molecular weight of the PCL precursor and crosslinking time $[14,17,19]$. Therefore, the swelling ratios increased from the range of 7.0-13.5 for PCLTA8k networks to 9.8-21.0 for PCLTA10k networks, and then, 17.5-32.8 for PCLTA20k networks. For the same PCLTA, the swelling ratio of the network decreased with increasing the crosslinking time. The crosslinking efficiency was higher for PCLTA8k and PCLTA10k than for PCLTA20k. A crosslinking time of $10 \mathrm{~min}$ was needed to reach the highest gel fractions of $94 \%$ for PCLTA8k and $92 \%$ for PCLTA10k, while a longer crosslinking time of $40 \mathrm{~min}$ was needed for PCLTA20k to reach the highest gel fractions of $80 \%$. When the crosslinking time increased from 1 to $3 \mathrm{~min}$, the gel fraction increased and the swelling ratio decreased dramatically for all the PCLTAs with different molecular weights. When the crosslinking time was longer than $10 \mathrm{~min}$, the increase in the gel fraction and the decrease in the swelling ratio of PCLTA8k and PCLTA10k could be barely noticeable, while the gel fraction increased and the swelling ratio decreased for PCLTA20k less sharply than the first $3 \mathrm{~min}$.
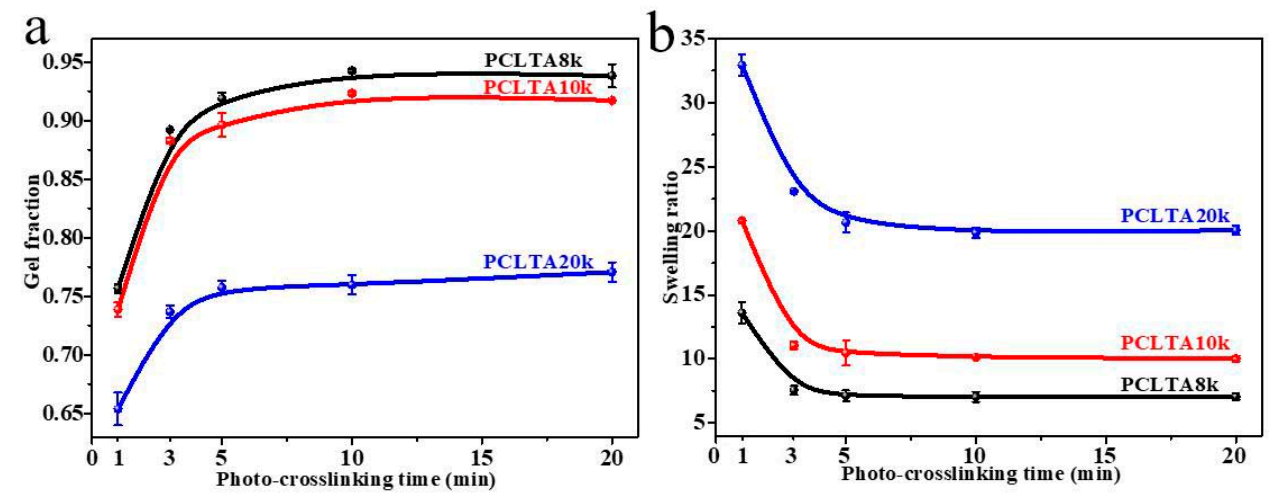

Figure 1. Gel fractions (a) and swelling ratios (b) of PCLTAs with different crosslinking time. 


\subsection{Thermal and Mechanical Properties}

The thermal properties $\left(\mathrm{T}_{\mathrm{m}}, \Delta \mathrm{H}_{\mathrm{m}}\right.$, and $\chi_{\mathrm{c}}$ ) of photo-crosslinked PCLTAs for various crosslinking times in Table 1 were obtained from the DSC curves in Figure 2. $\mathrm{T}_{\mathrm{m}}$ was the highest temperature of exothermal peaks during the heating round. $\chi_{c}$ was calculated using the equation of $\chi_{c}=\left[\Delta \mathrm{H}_{\mathrm{m}} /\left(\Phi_{\mathrm{PCL}} \cdot \Delta \mathrm{H}_{\mathrm{m}}{ }^{\mathrm{c}}\right)\right]$ $\times 100 \%$, where $\Delta \mathrm{H}_{\mathrm{m}}{ }^{\mathrm{c}}$ of completely crystalline PCL is $135 \mathrm{~J} / \mathrm{g}$ [20]. All uncrosslinked PCLTAs were semi-crystalline with higher $\mathrm{T}_{\mathrm{m}}$ and $\chi_{\mathrm{c}}$ than the crosslinked counterparts as the crosslinks suppressed crystallinity. Nevertheless, all the crosslinked PCLTAs were still semi-crystalline at the physiological temperature $\left(37^{\circ} \mathrm{C}\right)$. At the same crosslinking time, both $\mathrm{T}_{\mathrm{m}}$ and $\chi_{\mathrm{c}}$ increased from PCLTA8k and PCLTA10k to PCLTA20k. The $T_{m}$ of PCLTA10k was higher than that of PCLTA8k, while the difference in $\chi_{c}$ between PCLTA8k and PCLTA10k was insignificant. For the same PCLTA, $T_{m}$ and $\chi_{c}$ decreased dramatically when the crosslinking time increased from 0 to $3 \mathrm{~min}$. With increasing the crosslinking time further, $\chi_{\mathrm{c}}$ still decreased, but at a much lower rate, while $\mathrm{T}_{\mathrm{m}}$ only decreased slightly.

Table 1. Thermal properties of the crosslinked PCLTAs with different crosslinking time.

\begin{tabular}{ccccccccccc}
\hline \multirow{2}{*}{ Crosslinking Time (min) } & \multicolumn{3}{c}{$\mathbf{T}_{\mathbf{m}}\left({ }^{\circ} \mathbf{C}\right)$} & \multicolumn{3}{c}{$\mathbf{H}_{\mathbf{m}}(\mathbf{J} / \mathbf{g})$} & \multicolumn{3}{c}{$\chi_{\mathbf{c}}(\mathbf{\%})$} \\
\cline { 2 - 11 } & $\mathbf{8 k}$ & $\mathbf{1 0 k}$ & $\mathbf{2 0 k}$ & $\mathbf{8 k}$ & $\mathbf{1 0 k}$ & $\mathbf{2 0 k}$ & $\mathbf{8 k}$ & $\mathbf{1 0 k}$ & $\mathbf{2 0 k}$ \\
\hline 0 & 49.4 & 53.1 & 54.9 & 65.8 & 67.4 & 71.4 & 48.8 & 50.0 & 52.9 \\
1 & 41.4 & 44.0 & 51.3 & 52.2 & 51.4 & 60.7 & 38.7 & 38.0 & 45.0 \\
3 & 39.6 & 43.5 & 50.8 & 49.5 & 51.2 & 57.6 & 36.6 & 37.9 & 42.7 \\
5 & 39.5 & 43.3 & 50.9 & 49.0 & 51.0 & 57.2 & 36.3 & 37.8 & 42.4 \\
10 & 39.1 & 42.8 & 50.9 & 48.0 & 46.1 & 58.0 & 35.6 & 34.2 & 43.0 \\
20 & 39.0 & 42.7 & 50.7 & 42.5 & 45.5 & 56.5 & 31.5 & 33.7 & 41.9 \\
\hline
\end{tabular}
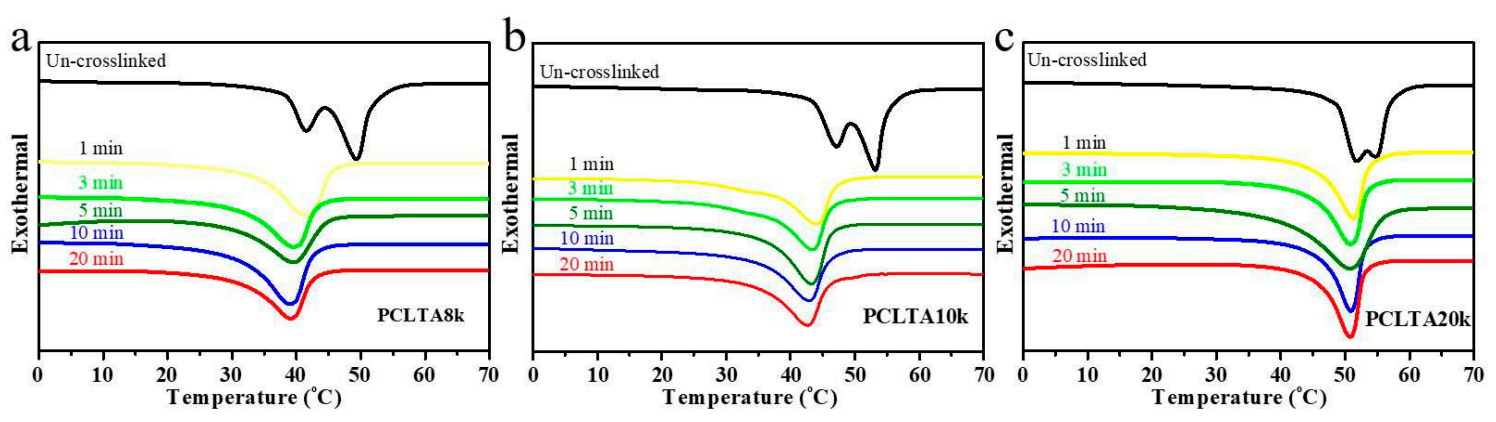

Figure 2. Differential scanning calorimeter (DSC) curves of crosslinked PCLTA8k (a), PCLTA10k (b), and PCLTA20k (c) with different crosslinking time.

Tensile testing was performed at $37^{\circ} \mathrm{C}$ to obtain the mechanical properties of photo-crosslinked PCLTAs at various crosslinking times. Representative stress-strain curves are shown in Figure 3. The $E$ increased from the range of 15.1-38.4 MPa for PCLTA8k to 37.9-128.5 MPa for PCLTA10k, and then 74.1-238.3 MPa for PCLTA20k. Because PCLTA20k had a higher $\chi_{c}$ than those of PCLTA8k and PCLTA10k, the stiffness increased with the increase of molecular weight at the same crosslinking time. Different from crosslinked PCLTAs, uncrosslinked PCLTAs were extremely brittle, with the strain at break of less than $10 \%$. For the same PCLTA, the stiffness increased dramatically with increasing the crosslinking time from 0 to $5 \mathrm{~min}$ (15.1 to $41.0 \mathrm{MPa}$ for PCLTA8k, 37.9 to $106.6 \mathrm{MPa}$ for PCLTA10k, and 74.1 to $210.1 \mathrm{MPa}$ for PCLTA20k), while the variance in stiffness was insignificant when the crosslinking time was longer than $10 \mathrm{~min}$ (41.0 to $38.4 \mathrm{MPa}$ for PCLTA8k, 106.6 to $128.5 \mathrm{MPa}$ for PCLTA10k, and 210.1 to $238.3 \mathrm{MPa}$ for PCLTA20k). The stiffness of crosslinked PCLTA20k always increased with increasing the crosslinking time, while the highest stiffness of crosslinked PCLTA8k and 10k was observed at the crosslinking time of 5 and $10 \mathrm{~min}$, respectively. 

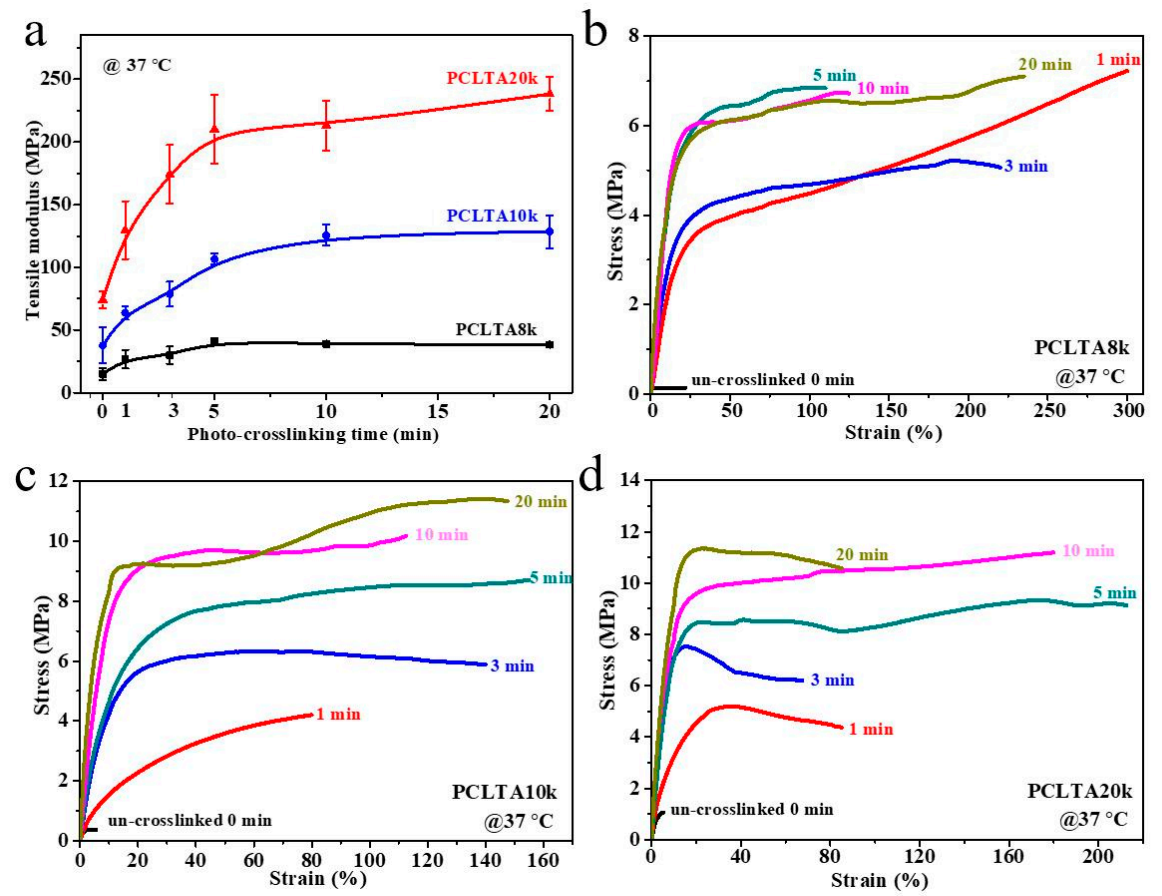

Figure 3. (a) Tensile modulus of PCLTAs (8k, 10k, and 20k) with different crosslinking time. Tensile stress-strain curves of PCLTAs ((b), 8k; (c), 10k; (d), 20k) with different crosslinking time.

Similar to uncrosslinked PCLTAs, crosslinked PCLTA disks at various crosslinking times were compressed and smoothened between two glass plates, and the surface morphologies were detected using AFM (Figure 4). After the compression, the $R_{\text {rms }}$ values of all the polymer disks were less than $20 \mathrm{~nm}$ without a significant difference. Therefore, the effect of surface roughness on cell behaviors could be neglected.

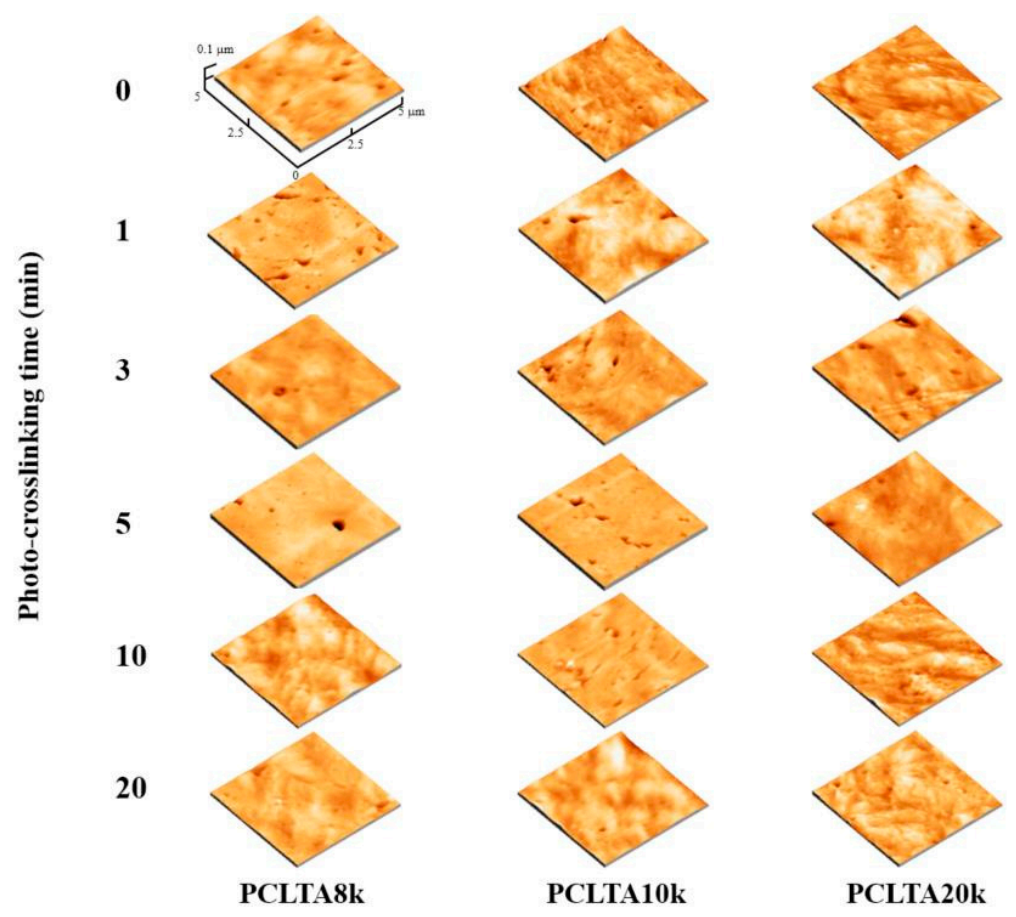

Figure 4. Atomic force microscope (AFM) 3D height images of PCLTAs with different crosslinking time. 


\subsection{In Vitro SMC Attachment and Proliferation}

SMCs were seeded on the crosslinked PCLTA disks at various crosslinking times, and the results of cell attachment, proliferation, and area are shown in Figure 5. Consistent with the findings in previous studies [25-28], stiffer substrates could support SMC attachment and proliferation better, indicated by those on photo-crosslinked PCLTA20k were better than on photo-crosslinked PCLTA8k and PCLTA10k at the same crosslinking time. Specifically, the difference in SMC proliferation became more significant when the crosslinking time was longer than $5 \mathrm{~min}$. For the same PCLTA, SMC attachment and proliferation were better when the crosslinking time was longer. Significant differences were found between the PCLTAs at crosslinking time longer than $10 \mathrm{~min}$ and shorter than $5 \mathrm{~min}$. Among the crosslinking time of $0,1,5$, and $20 \mathrm{~min}, \mathrm{SMC}$ attachment and proliferation had no significant difference on the same PCLTA networks. The cell images stained with RP and DAPI (Figure 6) were consistent with the cell numbers obtained using the MTS assay. SMC spread areas also followed the same trend as in cell attachment and proliferation. However, no significant difference was found on different PCLTAs at the same crosslinking time. For the same PCLTA, when the crosslinking time was higher than $5 \mathrm{~min}$, the increase in the cell area became more significant than on those at crosslinking time of $0,1 \mathrm{~min}$.

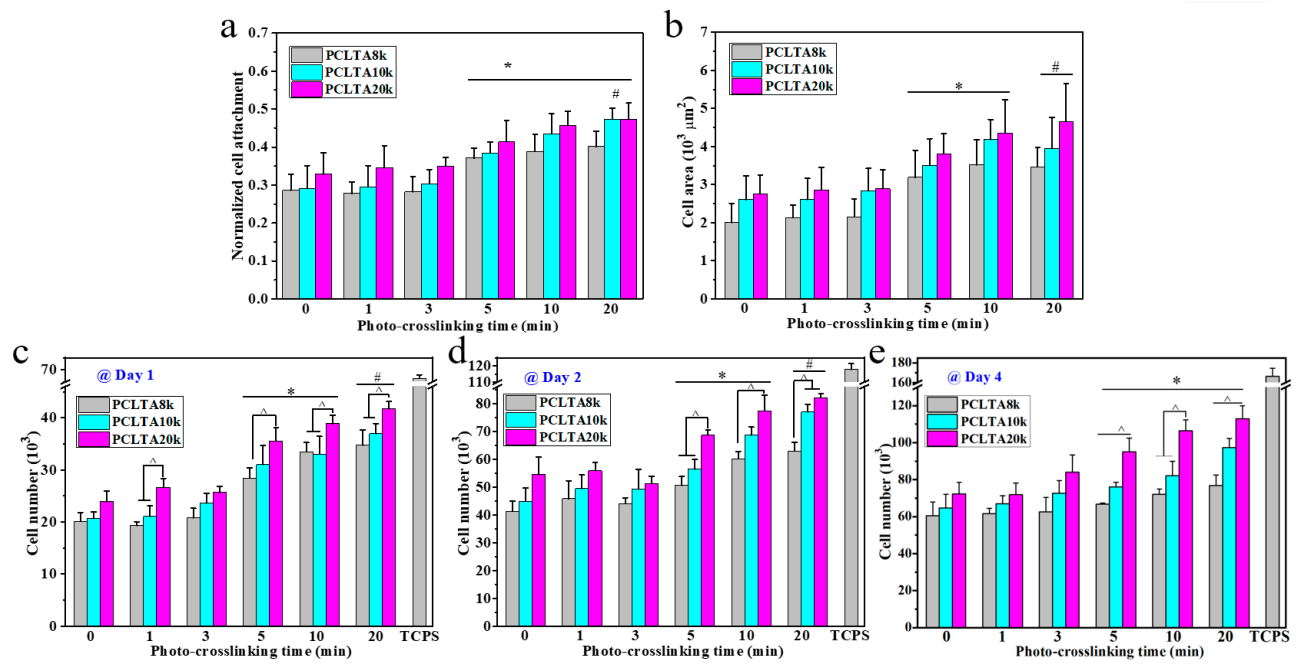

Figure 5. (a) Normalized smooth muscle cells (SMC) attachment at $4 \mathrm{~h}$ post seeding. (b) SMC areas at day 1 post-seeding. (c-e) SMC numbers at days 1, 2, and 4 post-seeding on the PCLTAs with different crosslinking time, respectively. ${ }^{*}: p<0.05$ between the samples marked with the symbol with the corresponding data on the same PCLTA with crosslinking time of 0,1 , and 3 min. ${ }^{\#}: p<0.05$ between the samples marked with the symbol with the corresponding data on the same PCLTA with crosslinking time of $0,1,3$, and 5 min. ${ }^{\prime}: p<0.05$ between the samples marked with the symbol.
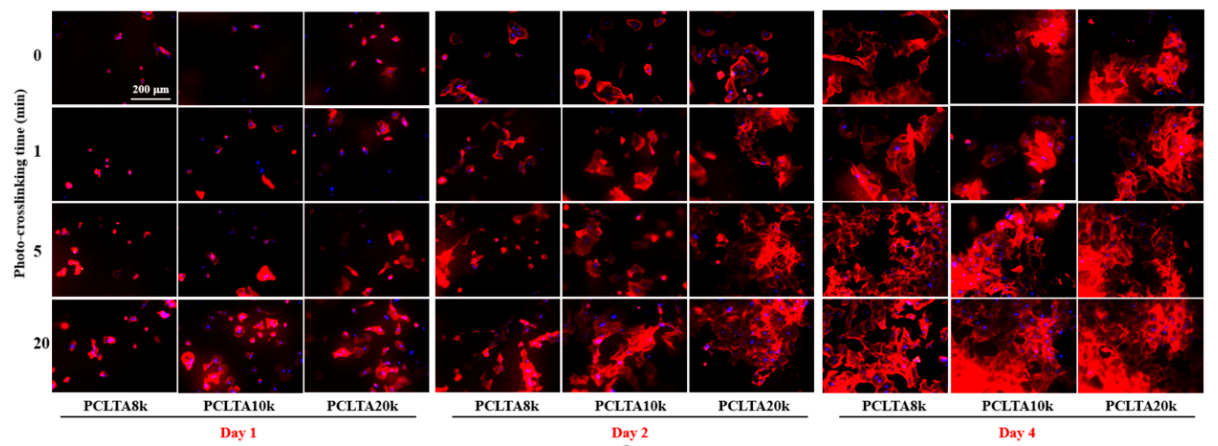

Figure 6. SMC images at days 1, 2, and 4 post-seeding stained using rhodamine-phalloidin (RP; red) and 4',6-diamidino-2-phenylindole (DAPI; blue) on PCLTAs with different crosslinking time. Scale bar of $200 \mu \mathrm{m}$ is applicable to all. 


\subsection{Focal Adhesions}

FAs are able to respond to external mechanical signals and adjust their own properties and simultaneously trigger mechanotransduction for further regulation of cell growth and spreading, and therefore, were used to further evaluate cell adhesion to the polymer substrates. FAs in the SMCs cultured for one day on the crosslinked PCLTA8k, 10k, and 20k disks at crosslinking time of 0, and 3 min were characterized and the results are shown in Figure 7. The FA density and area had the same trend as in the cell attachment and proliferation. For the same PCLTA, both FA density and area increased when the crosslinking time increased from 0 to $3 \mathrm{~min}$. At the same crosslinking time, FA density and FA area increased from PCLTA8k to PCLTA10k and then PCLTA20k; however, the difference was not significant among all the crosslinked PCLTAs at different crosslinking times.

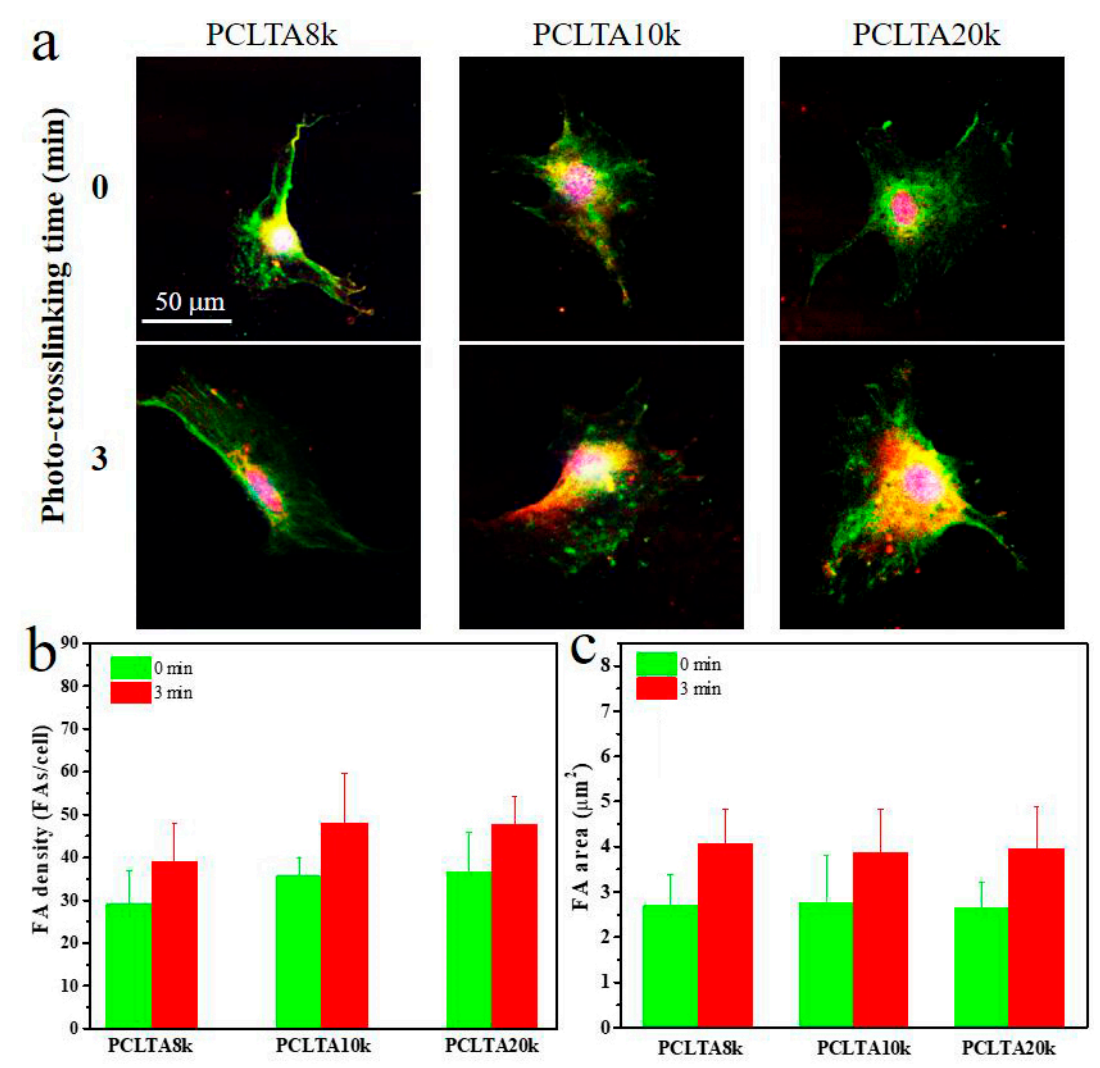

Figure 7. Characterizations of focal adhesions (FAs) in SMCs at day 1 post-seeding on PCLTAs with different crosslinking time (0,3 min). (a) Immunofluorescence images of FAs in the cells with vinculin stained green, and F-actin stained red. Scale bar of $50 \mu \mathrm{m}$ is applicable to all. Quantification of FAs in terms of (b) FA density, and (c) FA area.

\subsection{Further Discussion}

The present study attempted to understand how to achieve various thermal and mechanical properties through controlled crystallinity and crosslinking density by using PCLA networks that were crosslinked for different times. Without showing detectable cytotoxicity, all the PCLTA networks could support SMC adhesion, proliferation, and spreading. Meanwhile, the molecular weight and crosslinking density of PCLTAs resulted in different physical properties and, consequently, distinct SMC behaviors. Upon receiving the signals of the substrate materials, cells respond by adjusting their spreading, proliferation, and other related processes [29].

In terms of crosslinking efficiency, a difference was found between PCLTA20k and the other two PCLTAs with lower molecular weights. For PCLTA8k and PCLTA10k, crosslinking almost finished within 10 min of UV exposure, while for PCLTA20k, longer crosslinking time of 60 min could still increase the gel fraction and decrease the swelling ratio. The cell results showed the same trend with 
the gel fraction. With crosslinking time over $10 \mathrm{~min}$, the densities of SMCs on crosslinked PCLTA8k and PCLTA10k disks did not vary too much. In contrast, the cell density on PCLTA20k still increased, although this increase was insignificant.

The cell responses to different crosslinked PCLTA networks also provided the criteria of selecting biomaterials with sufficient crosslinking time to ensure appropriate crosslinking density and mechanical properties. PCLTAs were proven to have high crosslinking efficiency under UV exposure for only 5-10 min, and therefore, could be fabricated into two-dimensional substrates and three-dimensional scaffolds with better precision and controllability to fulfill different requirements for tissue engineering applications.

\section{Materials and Methods}

\subsection{Photo-Crosslinking and Characterization of PCLTAs}

Three PCLTAs with names of PCLTA8k, 10k, and 20k used in this study were synthesized according to a previous report and had number-average molecular weights $\left(M_{n}\right)$ of 8460,9750 , and 20,020 $\mathrm{g} / \mathrm{mol}$, respectively [18]. The PCLTAs were crosslinked into networks under UV light (SB-100P, $\lambda=365 \mathrm{~nm}$, Intensity: $4800 \mathrm{w} / \mathrm{cm}^{2}$ ) for different crosslinking times of 1, 3, 5, 10, and $20 \mathrm{~min}$, as shown in Figure 8 . The gel fraction and swelling ratio in $\mathrm{CH}_{2} \mathrm{Cl}_{2}$ of the crosslinked PCLTAs were determined according to a previous report [17]. Flat uncrosslinked PCLTA substrates (crosslinking time of 0 ) were prepared by compression and crystallization of the melt between two glass plates. Flat photo-crosslinked PCLTA substrates were soaked in acetone for two days to remove the sol fraction, dried in vacuum, and compressed between two glass plates. The thermal properties of the photo-crosslinked PCLTAs were performed using differential scanning calorimeter (DSC, Q2000, TA instruments). The samples were first heated from room temperature to $100{ }^{\circ} \mathrm{C}$ and then cooled to $-90^{\circ} \mathrm{C}$, followed by heating again to $100{ }^{\circ} \mathrm{C}$ at a rate of $10^{\circ} \mathrm{C} / \mathrm{min}$ in a dry nitrogen atmosphere. Linear viscoelastic properties of photo-crosslinked PCLTA disks $(8 \mathrm{~mm} \times 0.5 \mathrm{~mm}$, diameter $\times$ thickness $)$ were measured on a strain-controlled rheometer (RDS-2, Rheometric Scientific) at $37^{\circ} \mathrm{C}$. The surface morphologies of the photo-crosslinked PCLTAs were detected using a multi-mode atomic force microscope (AFM) with a Nanoscope III control system (Veeco Instruments, Santa Barbara, CA, USA). A tapping mode over a scan area of $5 \mu \mathrm{m} \times 5 \mu \mathrm{m}$ at a scan rate of $0.5 \mathrm{~Hz}$ was used. Root-mean-square surface roughness $\left(R_{\mathrm{rms}}\right)$ was measured from the height images using the Nanoscope 7.30 software (Veeco Instruments, Santa Barbara, CA, USA).
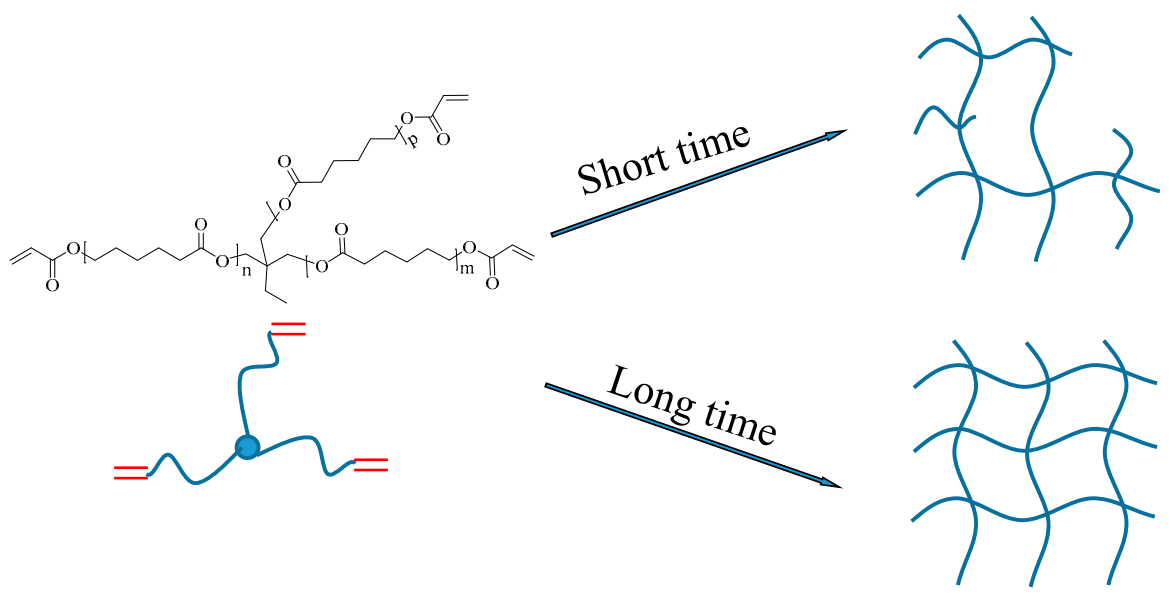

\section{PCLTA}

Photo-crosslinking

\section{Crosslinked PCLTA}

Figure 8. Scheme of the crosslinked poly( $\varepsilon$-caprolactone) acrylates (PCLTAs) with different crosslinking time. 


\subsection{In Vitro SMC Attachment and Proliferation}

Photo-crosslinked PCLTA disks were sterilized in 70\% ethanol solution and dried completely before cell studies. The primary aortic SMCs of Sprague-Dawley rats (Charles River, Wilmington, NC, USA), similar to humans' biological characteristics, were cultured in a growth medium composed of Dulbecco's modified eagle medium (DMEM; Gibco, Grand Island, NY, USA) supplemented with $10 \%$ fetal bovine serum (FBS; HyClone, Thermal Scientific, TX, USA) and 1\% penicillin/streptomycin (Gibco, Grand Island, NY, USA) in an incubator with $5 \% \mathrm{CO}_{2}$ and $95 \%$ relative humidity at $37^{\circ} \mathrm{C}$. SMCs were seeded onto PCLTA disks $(10 \times 0.5 \mathrm{~mm}$, diameter $\times$ thickness $)$ at a density of $15,000 \mathrm{cells} / \mathrm{cm}^{2}$ and cultured for $4 \mathrm{~h}, 1,2$, and 4 days. A colorimetric cell metabolic assay (CellTiter 96 Aqueous One Solution, Promega, Madison, WI, USA) was performed in each well to determine the number of attached cells, which was correlated with the UV absorbance of the solution at $490 \mathrm{~nm}$ measured on the microplate reader (SpectraMax Plus 384, Molecular Devices, Sunnyvale, CA, USA). Cell numbers were then quantified using the standard curve that was constructed using known cell numbers. SMCs attached on PCLTA disks were washed with phosphate buffered saline (PBS), fixed in $4 \%$ paraformaldehyde (PFA, Electron Microscopy Science, PA, USA) solution for $10 \mathrm{~min}$ at room temperature, washed with PBS twice, and then permeabilized with $0.1 \%$ Triton X-100 at room temperature for another $10 \mathrm{~min}$. The cytoplasm was then stained using rhodamine-phalloidin (RP, Cytoskeleton Inc., Denver, CO, USA) for $1 \mathrm{~h}$ at $37^{\circ} \mathrm{C}$, and cell nuclei were stained using $4^{\prime}, 6$-diamidino-2-phenylindole (DAPI) at room temperature. Then, the cells were photographed using an Axiovert 25 light microscope (Carl Zeiss, Göttingen, Germany). From the cell images, cell area was determined on more than 50 non-overlapping cells at day 1 by using the ImageJ software (National Institutes of Health, Bethesda, MD, USA).

\subsection{Focal Adhesions (FAs)}

After day 1 post-seeding, SMCs attached on the PCLTA disks with crosslinking times of 0 and 3 min were washed with PBS, fixed in $4 \%$ PFA solution, washed with PBS three times, and permeabilized with $0.1 \%$ Triton X-100 at room temperature for $10 \mathrm{~min}$. Then, the polymer disks with attached SMCs were incubated in $1 \%$ bovine serum albumin (BSA)/PBS at $37^{\circ} \mathrm{C}$ for $30 \mathrm{~min}$ to reduce the background. After being washed with PBS three times, the polymer diskes with cells were incubated in monoclonal mouse antibody against vinculin (1:1000 in 1\% PBS; Sigma, St. Louis, MO, USA) at room temperature for $2 \mathrm{~h}$, and then washed with PBS three times again. Then, the samples were cultured with goat anti-mouse IgG secondary antibody (1:200 in 1\% PBS; Sigma) in the dark at room temperature for $2 \mathrm{~h}$. The samples were also stained using RP at $37^{\circ} \mathrm{C}$ for another $1 \mathrm{~h}$. The FAs were photographed using a Leica DM6000B fluorescent confocal microscope. The density, area, and circularity (defined as $4 \pi \times$ area/perimeter ${ }^{2}$ ) of FAs were measured and averaged from 15 non-overlapping cells using ImageJ.

\subsection{Statistical Analysis}

The statistical difference among varied experimental groups was analyzed using Kruskal-Wallis with Mann-Whitney post hoc test. Any two data groups with p-value calculated lower than 0.05 were marked with a significant difference.

\section{Conclusions}

UV light have been applied to prepare cross-linkable PCLTA. The influences of crosslinking time on cellular behaviors of PCLTAs with different molecular weight were studied. A lower molecular weight and a longer crosslinking time resulted in a higher gel fraction, a lower swelling ratio, and a higher stiffness. Photo-crosslinking of PCLTAs largely was accomplished in the first 5 min of UV exposure. SMCs were cultured on these photo-crosslinked PCLTAs. At the same crosslinking time, PCLTA with a higher molecular weight could be crosslinked into substrates better supporting SMC attachment and proliferation. With the same molecular weight, better SMC attachment, proliferation, spreading, and focal adhesion were found on the photo-crosslinked PCLTA substrates at a longer 
crosslinking time. The increments in SMC attachment and proliferation were no longer significant after the crosslinking time was longer than $10 \mathrm{~min}$. Our photo-crosslinked PCLTA would be used to fabricate 3D scaffolds, demonstrating their potentials as biomaterials for diverse tissue-engineering applications.

Author Contributions: Writing—original draft, J.W.; data curation, L.L.; investigation, A.W.; methodology, software, X.L.; writing - review \& editing, Y.Z.; supervision, Z.W.; conceptualization, supervision, funding acquisition, J.D. All authors have read and agreed to the published version of the manuscript.

Funding: This research received no external funding.

Acknowledgments: The authors acknowledge support from United States National Science Foundation (DMR-11-06142 and DMR-15-07977), National Nature Science Foundation of China (51702157, 51902156), Youth Project of the Natural Science Foundation of Jiangsu Province, China (BK201710085). The authors are grateful to Shanfeng Wang for the technical support in experiment design and the help with manuscript preparation and revising.

Conflicts of Interest: The authors declare no conflict of interest.

\section{References}

1. Sharifi, F.; Atyabi, S.M.; Norouzian, D.; Zandi, M.; Irani, S.; Bakhshi, H. Polycaprolactone/carboxymethyl chitosan nanofibrous scaffolds for bone tissue engineering application. Int. J. Biol. Macromol. 2018, 115, 243-248. [CrossRef]

2. Rainbolt, E.A.; Washington, K.E.; Biewer, M.C.; Stefan, M.C. Recent developments in micellar drug carriers featuring substituted poly(E-caprolactone)s. Polym. Chem. 2015, 6, 2369-2381. [CrossRef]

3. Heydari, Z.; Mohebbi-Kalhori, D.; Afarani, M.S. Engineered electrospun polycaprolactone (PCL)/octacalcium phosphate (OCP) scaffold for bone tissue engineering. Mater. Sci. Eng. C 2017, 81, 127-132. [CrossRef] [PubMed]

4. Hatamzadeh, M.; Najafi-Moghadam, P.; Baradar-Khoshfetrat, A.; Jaymand, M.; Massoumi, B. Novel nanofibrous electrically conductive scaffolds based on poly (ethylene glycol) s-modified polythiophene and poly ( $\varepsilon$-caprolactone) for tissue engineering applications. Polymer 2016, 107, 177-190. [CrossRef]

5. Ahmed, M.K.; Mansour, S.F.; Al-Wafi, R.; Afifi, M.; Uskoković, V. Gold as a dopant in selenium-containing carbonated hydroxyapatite fillers of nanofibrous $\varepsilon$-polycaprolactone scaffolds for tissue engineering. Int. J. Pharm. 2020, 577, 118950. [CrossRef] [PubMed]

6. Turunen, M.P.K.; Korhonen, H.; Tuominen, J.; Seppälä, J.V. Synthesis, characterization and crosslinking of functional star-shaped poly(E-caprolactone). Polym. Int. 2002, 51, 92-100. [CrossRef]

7. Lendlein, A.; Schmidt, A.M.; Langer, R. AB-polymer networks based on oligo( $\varepsilon$-caprolactone) segments showing shape-memory properties. Proc. Natl. Acad. Sci. USA 2001, 98, 842.

8. Nagata, M.; Kanechika, M.; Sakai, W.; Tsutsumi, N. Biodegradable network elastomeric polyesters from multifunctional aromatic carboxylic acids and poly( $\varepsilon$-caprolactone) diols. J. Polym. Sci. Part A Polym. Chem. 2002, 40, 4523-4529. [CrossRef]

9. Nagata, M.; Kato, K.; Sakai, W.; Tsutsumi, N. Biodegradable network elastomeric polyesters from multifunctional aliphatic carboxylic acids and poly(epsilon-caprolactone) diols. Macromol. Biosci. 2006, 6, 333-339. [CrossRef]

10. Matsuda, T.; Mizutani, M.; Arnold, S.C. Molecular Design of Photocurable Liquid Biodegradable Copolymers. 1. Synthesis and Photocuring Characteristics. Macromolecules 2000, 33, 795-800. [CrossRef]

11. Han, C.; Ran, X.; Su, X.; Zhang, K.; Liu, N.; Dong, L. Effect of peroxide crosslinking on thermal and mechanical properties of poly( $\varepsilon$-caprolactone). Polym. Int. 2007, 56, 593-600. [CrossRef]

12. Zhu, G.; Xu, Q.; Qin, R.; Yan, H.; Liang, G. Effect of $\gamma$-radiation on crystallization of polycaprolactone. Radiat. Phys. Chem. 2005, 74, 42-50. [CrossRef]

13. Salgado, C.; Arrieta, M.P.; Peponi, L.; Fernández-García, M.; López, D. Silica-nanocomposites of photo-crosslinkable poly (urethane) s based on poly ( $\varepsilon$-caprolactone) and coumarin. Eur. Polym. J. 2017, 93, 21-32. [CrossRef]

14. Wang, S.; Yaszemski, M.J.; Gruetzmacher, J.A.; Lu, L. Photo-crosslinked poly( $\varepsilon$-caprolactone fumarate) networks: Roles of crystallinity and crosslinking density in determining mechanical properties. Polymer 2008, 49, 5692-5699. [CrossRef] [PubMed] 
15. Salgado, C.; Arrieta, M.P.; Peponi, L.; López, D.; Fernández-García, M. Photo-crosslinkable polyurethanes reinforced with coumarin modified silica nanoparticles for photo-responsive coatings. Prog. Org. Coat. 2018, 123, 63-74. [CrossRef]

16. Salgado, C.; Arrieta, M.P.; Sessini, V.; Peponi, L.; López, D.; Fernández-García, M. Functional properties of photo-crosslinkable biodegradable polyurethane nanocomposites. Polym. Degrad. Stab. 2020, 178, 109204. [CrossRef]

17. Cai, L.; Wang, S. Poly( $\varepsilon$-caprolactone) acrylates synthesized using a facile method for fabricating networks to achieve controllable physicochemical properties and tunable cell responses. Polymer 2010, 51, 164-177. [CrossRef]

18. Chiappelli, M.C.; Hayward, R.C. Photonic Multilayer Sensors from Photo-Crosslinkable Polymer Films. Adv. Mater. 2012, 24, 6100-6104. [CrossRef]

19. Wang, K.; Cai, L.; Wang, S. Methacryl-polyhedral oligomeric silsesquioxane as a crosslinker for expediting photo-crosslinking of Poly(propylene fumarate): Material properties and bone cell behavior. Polymer 2011, 52, 2827-2839. [CrossRef]

20. Wang, S.; Kempen, D.H.; Simha, N.K.; Lewis, J.L.; Windebank, A.J.; Yaszemski, M.J.; Lu, L. Photo-Cross-Linked Hybrid Polymer Networks Consisting of Poly(propylene fumarate) and Poly(caprolactone fumarate): Controlled Physical Properties and Regulated Bone and Nerve Cell Responses. Biomacromolecules 2008, 9, 1229-1241. [CrossRef]

21. Zhu, J. Bioactive modification of poly(ethylene glycol) hydrogels for tissue engineering. Biomaterials 2010, 31, 4639-4656. [CrossRef] [PubMed]

22. Liu, Q.; Jiang, L.; Shi, R.; Zhang, L. Synthesis, preparation, in vitro degradation, and application of novel degradable bioelastomers-A review. Prog. Polym. Sci. 2012, 37, 715-765. [CrossRef]

23. Venhoven, B.A.M.; de Gee, A.J.; Davidson, C.L. Light initiation of dental resins: Dynamics of the polymerization. Biomaterials 1996, 17, 2313-2318. [CrossRef]

24. Finch, C.A. Polymer handbook: Third edition Edited by J. Brandrup and E. H. Immergut, Wiley-Interscience, Chichester, 1989. pp. ix + parts I to VIII, price $£ 115 \cdot 00 / \$ ; 175 \cdot 00$. ISBN 0-471-81244-7. Br. Polym. J. 1990, 23, 277. [CrossRef]

25. Wong, J.Y.; Velasco, A.; Rajagopalan, P.; Pham, Q. Directed Movement of Vascular Smooth Muscle Cells on Gradient-Compliant Hydrogels. Langmuir 2003, 19, 1908-1913. [CrossRef]

26. Dennis, E.; Discher, P.J.; Wang, Y. Tissue Cells Feel and Respond to the Stiffness of Their Substrate. Science 2005, 310, 1139-1143.

27. McDaniel, D.P.; Shaw, G.A.; Elliott, J.T.; Bhadriraju, K.; Meuse, C.; Chung, K.-H.; Plant, A.L. The Stiffness of Collagen Fibrils Influences Vascular Smooth Muscle Cell Phenotype. Biophys. J. 2007, 92, 1759-1769. [CrossRef]

28. Isenberg, B.C.; DiMilla, P.A.; Walker, M.; Kim, S.; Wong, J.Y. Vascular Smooth Muscle Cell Durotaxis Depends on Substrate Stiffness Gradient Strength. Biophys. J. 2009, 97, 1313-1322. [CrossRef]

29. Jiang, F.X.; Yurke, B.; Schloss, R.S.; Firestein, B.L.; Langrana, N.A. The relationship between fibroblast growth and the dynamic stiffnesses of a DNA crosslinked hydrogel. Biomaterials 2010, 31, 1199-1212. [CrossRef]

Publisher's Note: MDPI stays neutral with regard to jurisdictional claims in published maps and institutional affiliations.

(C) 2020 by the authors. Licensee MDPI, Basel, Switzerland. This article is an open access article distributed under the terms and conditions of the Creative Commons Attribution (CC BY) license (http://creativecommons.org/licenses/by/4.0/). 